\title{
THE POLARIZATION OF RADIATION IN SINGLE CRYSTALS IN THE QUASICLASSICAL APPROACH
}

\author{
S.M.Darbinyan ${ }^{a}$ and N.L.Ter-Isaakyan ${ }^{b}$ \\ Yerevan Physics Institute Yerevan 375036, Armenian \\ ${ }^{a}$ E-mail: simon@lx2.yerphi.am \\ ${ }^{b}$ E-mail: terisaak@jerewan1.yerphi.am
}

\begin{abstract}
The radiation emission spectra of polarized photons emitted from charged particles in single oriented crystals are obtained in Bayer-Katkov quasiclassical approach. The results of numerical calculations are presented in the region of small angles of incidence for which the coherent theory fails but magnetic bremsstrahlung region isn't yet achieved. The spectral distribution of linear polarization degree repeats, in general, the form of the intensity distribution. At sufficiently small angles, in the case of planar orientation of crystal, there is a wide maximum of essential linear polarization at low frequencies $\omega / \varepsilon=0.1-0.3$.
\end{abstract}

The processes of photon emission from charged particles and $e^{-} e^{+}$pair creation by photon at high energies in oriented single crystals are widely applied in experimental physics for the production of polarized photon beams, as well as for the analyses of photon polarization. For sufficiently large incident angles $\vartheta_{0}$ to crystal axis/planes $\vartheta_{0} \gg \vartheta_{v}$, where $\vartheta_{v}$ is the characteristic angle given by $\vartheta_{v}=U_{0} / m\left(U_{0}\right.$ is the scale of axial/planar potential and $m$ the electron mass) these processes are well described in the theory of coherent bremsstrahlung (CB) and pair production (CPP) [1]. This theory is constructed in the framework of the first Born approximation in crystal potential and fails at small angles and very high energies, where these processes become of magnetic bremsstrahlung nature.

Recently V.N. Bayer with co-authors developed a general theory for radiation emission from high-energy particles and pair production by high-energy photons in strong crystalline fields [2]. This theory is not restricted by the first Born approximation and is based on the quasiclassical character of motion of ultrarelativistic particles in strong fields. For $\vartheta_{0} \gg \vartheta_{v}$, this theory is reduced to the standard theory for CB and CPP. At very small angles $\vartheta_{0} \ll \vartheta_{v}$, the quasiclassical theory reproduces the results of constant field approximation (CFA). For intermediate angles, $\vartheta_{0} \sim \vartheta_{v}$, the numerical calculations in the exact theory face serious mathematical difficulties and to obtain specific numerical results some approximation and modeling methods have been developed. First numerical results of the radiation emission spectra in the quasiclassical theory were published in [3] (in the framework of additional modeling assumption); exact calculations were presented in [4]. In Ref. [5] an analytical method of calculations in the framework of quasiclassical approach was developed and new numerical results were presented. These papers demonstrate an essential difference of exact spectra from the corresponding results of coherent theory at small angles $\vartheta_{0} \leq \vartheta_{v}$. There is good agreement between first experimental results at small angles $[6,7]$ and these calculations.

Polarization characteristics of radiation emission in single crystals in the region of applicability of CB are well investigated in experiments (at the energies of incident electrons up to

\footnotetext{
${ }^{1}$ The work is supported by ISTC grant A-099
} 
$10 \mathrm{GeV}$ ) and the CB theory describes satisfactory the experimental results [1].

It is very important to investigate the polarization phenomena at very high energies in oriented crystals, where the CB theory doesn't work. If we could find optimal crystal orientations, which bring to the large intensity enhancements of essentially polarized photons at large frequencies, it will give us an interesting possibility of producing high energy polarized photon beams. However, at energies of $100 \mathrm{GeV}$ and higher the polarization characteristics of radiation emission have not yet been investigated experimentally and there are no publications presenting numerical calculations in the quasiclassical theory.

In this paper the formulae for radiation emission spectra of polarized photons are derived in Bayer-Katkov quasiclassical approach. We also present preliminary results of numerical calculations in the most interesting region of intermediate angles $\vartheta_{0} \leq \vartheta_{v}$ for planar orientation of crystal, where maximal polarization effects are expected.To carry out the arising integrals over time of complicated oscillating functions we have elaborated the calculation algorithm and the special integration program, which is very effective especially at small angles.

We start from the general Bayer-Katkov quasiclassical formula [2] which gives the spectral distribution of polarized photon energy, averaged over the initial and summed over the final electron polarizations:

$$
\begin{gathered}
d E=\alpha \frac{d^{3} k}{(2 \pi)^{2}} \int_{-\infty}^{\infty} d t_{1} \int_{-\infty}^{\infty} d t_{2} \frac{1}{4 \varepsilon^{\prime 2}}\left[\left(\varepsilon+\varepsilon^{\prime}\right)^{2}\left(\mathbf{e v}_{2}\right)\left(\mathbf{e}^{*} \mathbf{v}_{1}\right)-\omega^{2}\left(\mathbf{e v}_{1}\right)\left(\mathbf{e}^{*} \mathbf{v}_{2}\right)\right. \\
\left.+\omega^{2}\left(\mathbf{v}_{1} \mathbf{v}_{2}-1+\frac{1}{\gamma^{2}}\right)\left(\mathbf{e e}^{*}\right)\right] e^{i k^{\prime}\left(x_{1}-x_{2}\right)}
\end{gathered}
$$

where $\alpha=1 / 137$ is fine structure constant, $k^{\mu}=(\omega, \mathbf{k})$ and $\mathbf{e}$ - stands, correspondingly, for photon 4-momenta and polarization vector; $\varepsilon, \varepsilon^{\prime}=\varepsilon-\omega$ - stands for initial and final electron energies, $k^{\prime \mu}=k^{\mu} \varepsilon / \varepsilon^{\prime}, \gamma=\varepsilon / m ; \mathbf{r}_{1,2}=\mathbf{r}\left(t_{1,2}\right)$ and $\mathbf{v}_{1,2}=\mathbf{v}\left(t_{1,2}\right)$ are electron coordinates and velocities at the moment of time $t_{1,2}, x_{1,2}=\left(t_{1,2}, \mathbf{r}_{1,2}\right)$.

We are going to find the radiation emission spectra of polarized photons, integrated over the emitted photon angles $\vartheta, \varphi$. Therefore, the photon polarization vectors must be defined via the direction which remains fixed (keeping in mind smallness of $\vartheta, \vartheta \leq 1 / \gamma$ ) after integration over $\vartheta, \varphi$. We chose two independent polarization vectors in the following form:

$$
\mathbf{e}_{1}=\frac{\left[\mathbf{n}_{2} \mathbf{n}\right]}{\left|\left[\mathbf{n}_{2} \mathbf{n}\right]\right|}, \mathbf{e}_{2}=\left[\mathbf{n e}_{1}\right]
$$

where $\mathbf{n}=\mathbf{k} / \omega, \mathbf{n}_{2}$ is a unit vector transverse to the incident electron velocity $\mathbf{v}_{0}$, the exact directions of $\mathbf{n}_{2}$ can be fixed by experimental layout. We choose $\mathbf{n}_{2}$ transverse to the crystal axis, along which the incident electron is aligned. At $\vartheta \ll 1$ polarization vectors $\mathbf{e}_{1}$ and $\mathbf{e}_{2}$ have the same fixed directions for any $\varphi$, and the Stocks parameters defined in this vectors, correspond to the definite directions after integration over emitted photon angles.

Utilizing the method derived in ref.[2], we integrate (1) over photon angles $\vartheta, \varphi$ and go over to the intensity $I=d E / d t$. If longitudinal length of crystal is much higher than radiation formation length, after averaging over electron trajectories in crystal, we can neglect the intensity dependence on time [2]. Finally, we present the polarized photon emission spectra in terms of Stocks parameters $\xi_{1}, \xi_{2}, \xi_{3}$ :

$$
\frac{d I}{d \omega}=\frac{\alpha}{\pi} \frac{m^{2} \omega}{\varepsilon^{2}} \int \frac{d^{3} r_{0}}{V} F\left(\mathbf{r}_{\mathbf{0}}, \vartheta_{0}\right)\left[\int_{0}^{\infty} \frac{d \tau}{\tau}\left[\left(T_{0}+\gamma^{2} \xi_{1} T_{1}+\gamma^{2} \xi_{3} T_{3}\right) \sin A_{1}+\gamma^{2} \xi_{2} T_{2}\right]-\frac{\pi}{2}\right]
$$


Where $\mathbf{r}_{0}$ is the entry point, $V$ is crystal volume, integration over $\mathbf{r}_{0}$ gives the averaging over electron trajectories in crystal, $F\left(\mathbf{r}_{0}, \vartheta_{0}\right)$ is the coordinate distribution function at given value of $\vartheta_{0}$. The quantities of $A_{1}=A_{1}(\tau)$ and $T_{i}=T_{i}(\tau), i=0,1,2,3$, in (3) are defined as follows:

$$
\begin{gathered}
A_{1}=\frac{m^{2} \omega}{2 \varepsilon \varepsilon^{\prime}}\left[1+\gamma^{2}\left[\frac{1}{\tau} \int_{-\tau / 2}^{\tau / 2} \mathbf{v}_{\perp}^{2}(t) d t-\mathbf{a}_{\perp}{ }_{\perp}(\tau)\right]\right] \\
T_{0}=1+\frac{1}{4} \varphi(\varepsilon) \gamma^{2}\left(\mathbf{v}_{1 \perp}-\mathbf{v}_{2 \perp}\right)^{2} \\
T_{1}=-2 a_{x} a_{y}+a_{y}\left(v_{1 x}+v_{2 x}\right)+a_{x}\left(v_{1 y}+v_{2 y}\right)-\left(v_{1 x} v_{2 y}+v_{1 y} v_{2 x}\right) \\
T_{2}=\frac{\varphi(\varepsilon)}{2}\left(\mathbf{a}\left[\mathbf{v}_{1} \mathbf{v}_{2}\right]\right) \\
T_{3}=a_{y}^{2}-a_{x}^{2}+a_{x}\left(v_{1 x}+v_{2 x}\right)-a_{y}\left(v_{1 y}+v_{2 y}\right)-\left(v_{1 x} v_{2 x}-v_{1 y} v_{2 y}\right)
\end{gathered}
$$

where $\mathbf{a}(\tau)=\frac{1}{\tau} \int_{-\tau / 2}^{\tau / 2} \mathbf{v}(t) d t, \quad \varphi(\varepsilon)=\frac{\varepsilon^{\prime}}{\varepsilon}+\frac{\varepsilon}{\varepsilon^{\prime}}, \quad \mathbf{v}_{\perp}=\left(v_{x}, v_{y}\right), \mathbf{v}_{1}=\mathbf{v}(-\tau / 2), \quad \mathbf{v}_{2}=\mathbf{v}(\tau / 2)$.

The Stocks parameters and projections of vectors correspond to the frame defined by following unit vectors:

$$
\hat{e}_{x}=\left[\mathbf{n}_{2} \mathbf{v}_{0}\right], \hat{e}_{y}=\mathbf{n}_{2}, \hat{e}_{z}=\mathbf{v}_{0}
$$

i.e. $v_{x}=\mathbf{v} \hat{e}_{x}, v_{y}=\mathbf{v} \hat{e}_{y}$ and so on.

The formulae for polarized photon emission spectra in quasiclassical approach were derived earlier in ref.[2] and an example of numerical calculations (in the range of applicability of modified coherent theory) was presented in [3]. Our results for unpolarized photon $\left(T_{0}\right)$ and circular polarized photon $\left(T_{2}\right)$ coincide with [2]. For linear polarization $\left(T_{1}\right.$ and $\left.T_{3}\right)$ our formulae are completely different and the results of our numerical calculations (see below) differ from [3] even qualitatively! The difference is originated in the choice of the photon polarization vectors. In ref.[2] the independent photon polarization vectors were chosen, correspondingly, in the reaction plane and transverse to it. At such a choice, if we are interested in the polarization characteristics of emitted photon, we should not immediately integrate the polarized spectra over azimuth angle $\varphi$, because after such integration we have no fixed direction perpendicular to incident electron velocity, and therefore the Stocks parameters cannot be connected with any fixed direction in space. One can see that in such calculations even the quantity $P=\sqrt{\xi_{1}^{2}+\xi_{2}^{2}}$ cannot be associated with the degree of linear polarization of emitted photon. For instance, the value of $\mathrm{P}$ carried out in such a way for amorphous medium does not vanish after integration over $\varphi$ !

For further calculations we shall use the rectilinear trajectory approximation which is valid, strongly speaking, for $\vartheta_{0} \gg \vartheta_{c}$, where $\vartheta_{c}$ is the Lindhard channeling angle [8]. Substituting into (4)-(8) the electron transverse velocity in crystal in rectilinear trajectory approximation, we finally find the following equations for the quantities $A_{1}, T_{i}$ which determine the polarized photon emission spectra of Eq.(3):

$$
A_{1}=\frac{m^{2} \omega \tau}{\varepsilon \varepsilon^{\prime}}\left[1+\sum_{\mathbf{q}, \mathbf{q}^{\prime}} \frac{G(\mathbf{q}) G\left(\mathbf{q}^{\prime}\right)}{m^{2} q_{z} q_{z}^{\prime}}\left(\mathbf{q}_{\perp} \mathbf{q}_{\perp}^{\prime}\right)\left[\frac{\sin \left(\left(q_{z}+q_{z}^{\prime}\right) \tau\right)}{\left(q_{z}+q_{z}^{\prime}\right) \tau}-\frac{\sin \left(q_{z} \tau\right) \sin \left(q_{z}^{\prime} \tau\right)}{q_{z} q_{z}^{\prime} \tau^{2}}\right] e^{-i\left(\mathbf{q}+\mathbf{q}^{\prime}\right) \mathbf{r}}\right]
$$




$$
\begin{gathered}
T_{0}=\left[1-\varphi(\varepsilon) \sum_{\mathbf{q}, \mathbf{q}^{\prime}} \frac{G(\mathbf{q}) G\left(\mathbf{q}^{\prime}\right)}{m^{2} q_{z} q_{z^{\prime}}}\left(\mathbf{q}_{\perp} \mathbf{q}_{\perp}^{\prime}\right) \sin \left(q_{z} \tau\right) \sin \left(q_{z}^{\prime} \tau\right) e^{-i\left(\mathbf{q}+\mathbf{q}^{\prime}\right) \mathbf{r}}\right], \\
T_{1}=-\sum_{\mathbf{q}, \mathbf{q}^{\prime}} \frac{G(\mathbf{q}) G\left(\mathbf{q}^{\prime}\right)}{m^{2} q_{z} q_{z}^{\prime}}\left(q_{x} q_{y}^{\prime}+q_{y} q_{x}^{\prime}\right) e^{-i\left(\mathbf{q}+\mathbf{q}^{\prime}\right) \mathbf{r}}\left[g\left(q_{z} \tau\right) g\left(q_{z}^{\prime} \tau\right)+\sin \left(q_{z} \tau\right) \sin \left(q_{z}^{\prime} \tau\right)\right], \\
T_{2}=\varphi(\varepsilon) \sum_{\mathbf{q}, \mathbf{q}^{\prime}} \frac{G(\mathbf{q}) G\left(\mathbf{q}^{\prime}\right)}{m^{2} q_{z} q_{z}^{\prime}}\left(q_{x} q_{y}^{\prime}-q_{y} q_{x}^{\prime}\right) e^{-i\left(\mathbf{q}+\mathbf{q}^{\prime}\right) \mathbf{r}}\left[g\left(q_{z} \tau\right) \sin \left(q_{z}^{\prime} \tau\right)-g\left(q_{z}^{\prime} \tau\right) \sin \left(q_{z} \tau\right],\right. \\
T_{3}=-\sum_{\mathbf{q}, \mathbf{q}^{\prime}} \frac{G(\mathbf{q}), G\left(\mathbf{q}^{\prime}\right)}{m^{2} q_{z} q_{z}^{\prime}}\left(q_{x} q_{x}^{\prime}-q_{y} q_{y}^{\prime}\right) e^{-i\left(\mathbf{q}+\mathbf{q}^{\prime}\right) \mathbf{r}}\left[g\left(q_{z} \tau\right) g\left(q_{z}^{\prime} \tau\right)+\sin \left(q_{z} \tau\right) \sin \left(q_{z}^{\prime} \tau\right)\right], \\
g(x)=\frac{\sin x}{x}-\cos x .
\end{gathered}
$$

The notation used here coincide, in general, with ones of ref.[2], $\mathbf{q}$ is reciprocal lattice vector; the crystal potential is used in the form of $U(r)=\sum G(\mathbf{q}) e^{-i \mathbf{q r} r}$. In numerical calculation we use the Moliere potential. At large angles Eqs.(3), (10)-(14) turn into the well known equations of polarized photon emission spectra of CB theory [1].

We have carried out the numerical calculations at $\varepsilon=150 \mathrm{GeV}$ for $<001>$ aligned single diamond crystal at temperature $T=293 K$, $\vartheta_{0}$ is the angle of the vector $\mathbf{v}_{0}$ to this axis, and $\varphi_{0}$ is the angle of the $\mathbf{v}_{0}$ projection onto the plane $\{001\}$ to the plane $\{1 \overline{1} 0\}$. The calculations are carried out for uniform distribution. On Fig.1, 2 the graduate changing of intensity and polarization spectra are presented when the angle $\psi$ of the vector $\mathbf{v}_{0}$ to the plane $\{1 \overline{1} 0\} \quad\left(\sin \psi=\sin \vartheta_{0} \sin \varphi_{0}\right)$ is decreasing. The behavior of the unpolarized intensity coincides, in general, with results of ref.4. The shape of the spectra is determined by the competition of coherent effects, which are determined by high time region $\tau \geq 1 / q_{z}$ in the integrals over time in (3), and magnetic bremsstrahlung effects, which come

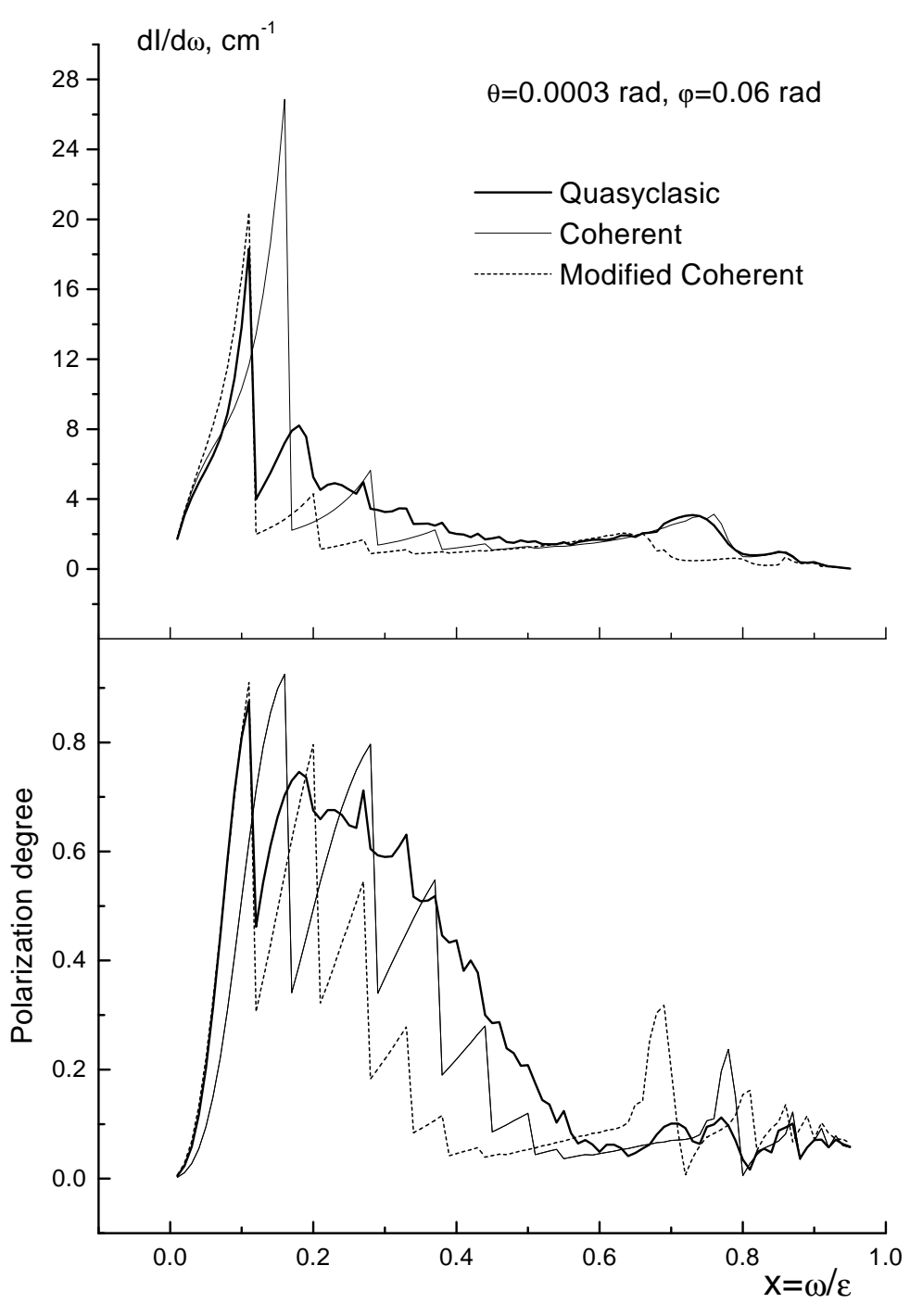

Fig.1: Comparison the spectral distributions of intensity and degree of polarization in coherent [1] and quasiclassical [2] approaches at relatively large angles. 
from low time region $\tau \ll 1 / q_{z}$. As

compared with the $\mathrm{CB}$ results, the

intensity of coherent peaks decreases, the peaks change their form and move down to low frequencies $\omega$. These changes are partly stipulated by renormalization of electron mass in strong crystalline fields. One can also see the arising of new peaks and deeps [4], which also move to low $\omega$ region. At the same time, when $\psi$ decreases, magnetic bremsstrahlung effects begin to contribute and bring to overall increasing of intensity. They are stronger displayed at higher electron energies and lower radiated frequencies, because the effective upper integration limit in $(3), \tau_{0} \geq c \cdot \varepsilon \varepsilon^{\prime} / m^{2} \omega,(c \gg 1)$, increases with increasing of the electron energy and with decreasing of the photon energy.

In the wide range of angles the radiation has an essential linear polarization. The circular polarization doesn't vanish but practically is negligible $\left(\xi_{2} \leq 0.01\right)$. As in the CB theory, the spectral distribution of the degree of polarization repeats, in general, the structure of the intensity distribution, i.e. peaks of intensity and peaks of polarization are situated at the same frequencies. So our results differ qualitatively from the results of ref.3, where at the positions of the intensity maxima are situated the minima of polarization!

When $\varphi_{0}$ is decreasing at fixed $\vartheta_{0}$ (Fig.2) (transfer to purely planar orientation), vectors $q_{z}$ which contribute to sums in Eq.[3], form different groups, in such a way, that each vector $q_{z}$ from the given group tends to the same limit at $\varphi_{0} \rightarrow 0$, $q_{z} \rightarrow q_{z}^{(i)}, \quad(\mathrm{i}=1,2,3, \ldots) . \quad$ The first group of vectors which tend to zero, $q_{z}^{(i)}=0$, determine the magnetic bremsstrahlung contribution in the range of small $\omega$. This contribution brought to the large maximum of the degree of linear polarization (0.6-0.8) at $x=\omega / \varepsilon=0.1 \div 0.3$, which remains unchanged at further decrease of $\varphi_{0}$. The radiation is polarized in $\{110\}$ plane $\left(\xi_{1} \sim 0\right)$. The

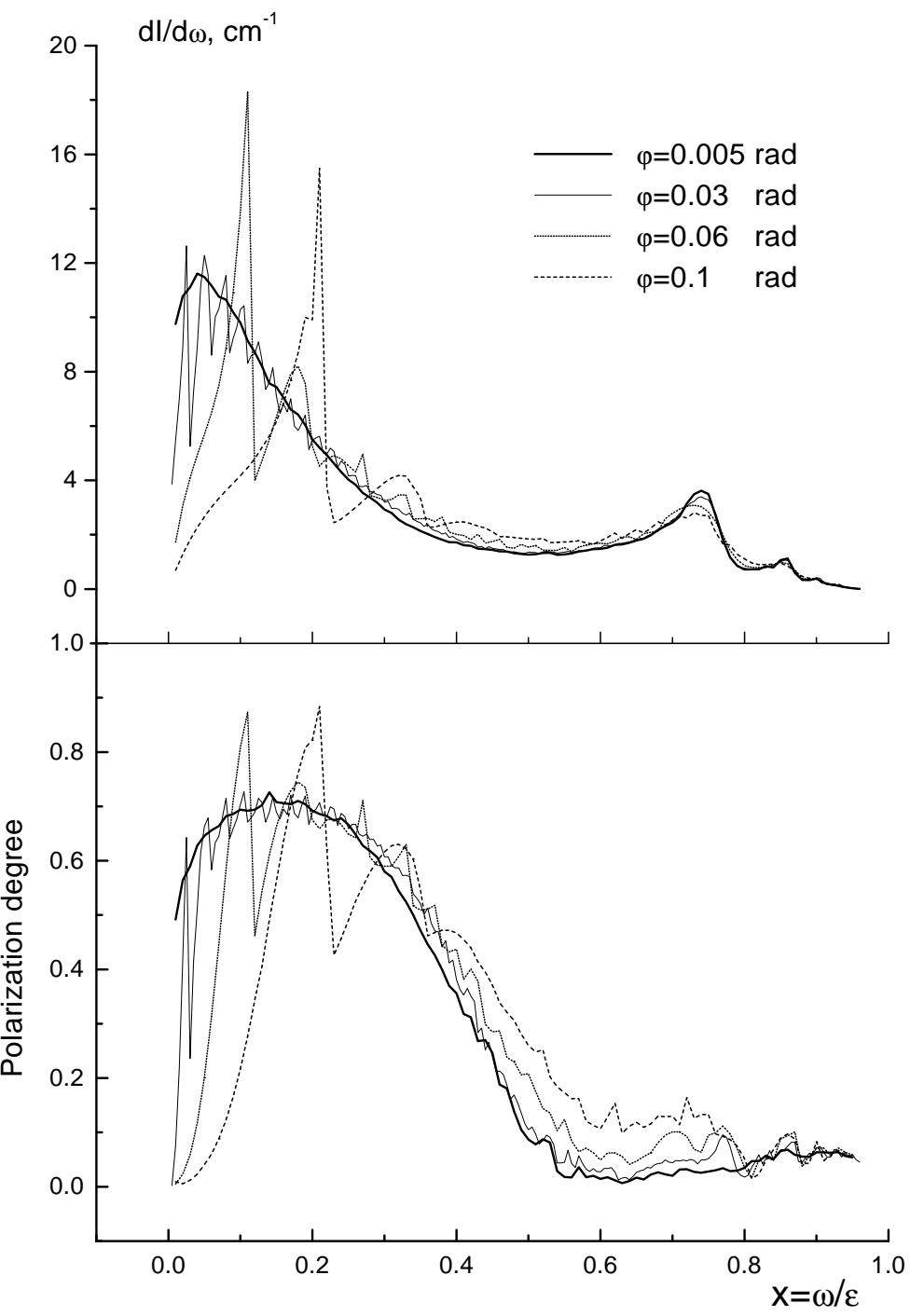

Fig.2: Changes of intensity and polarization spectra at decreasing of $\varphi_{0}$ at fixed $\vartheta_{0}=0.3 \mathrm{mrad}$. groups of the vectors, which tend to nonvanishing limits, $\left(q_{z}^{(i)} \neq 0\right)$ correspond to a number of high frequency peaks. If these peaks are situated at sufficiently large frequencies $(x \geq 0.6)$, the magnetic bremsstrahlung effects 
are small enough and do not affect seriously the formation of these peaks, so the shape and position of these peaks can be approximately described in (non modified) coherent theory. The magnitude of the peaks is determined, approximately, as a sum of coherent contributions of reciprocal vectors with given $q_{z}^{(i)}$. For the intensities of polarized photon emission, these contribution could be of different sign, or some contribution could vanish. Therefore, the polarization in the region of high $\omega$ peaks strongly depends on crystal orientation and is relatively small. For instance, for $<001>$ aligned crystal, in the region of main high frequency peak, the individual contributions practically compensate each other and degree of polarization is very small. For $<110>$ orientation, in the range of similar peak the main contributions appear to be of the same sign, and degree of polarization reaches 0.25 (Fig.3).

Let us also note that, despite the planar orientation of crystal, the direction of polarization at these peaks is not immediately connected with the position of main plane. We have, in general, $\left|\xi_{1}\right| \sim\left|\xi_{3}\right| . \quad$ It is easy to understand, because according to what was explained above, the main plain practically do not contributions to these peaks, contributions come only from neighbouring planes, which are situated at different angles to the main plane. The "fine structure" of the spectral distribution of intensity and Stocks parameters for different crystal orientations in the range of high frequency peak are demonstrated in Fig.3.

High-energy photon pronounced maxima in the radiation emission have been observed experimentally at CERN [6] in diamond and $\mathrm{Si}$ crystals. A discussion is going on as to the possibility of utilizing this effect for producing high-energy polarized photon beams.

More precise calculations for different crystal orientations in the wide range of incident electron angles and energies we are going to present in subsequent pa-

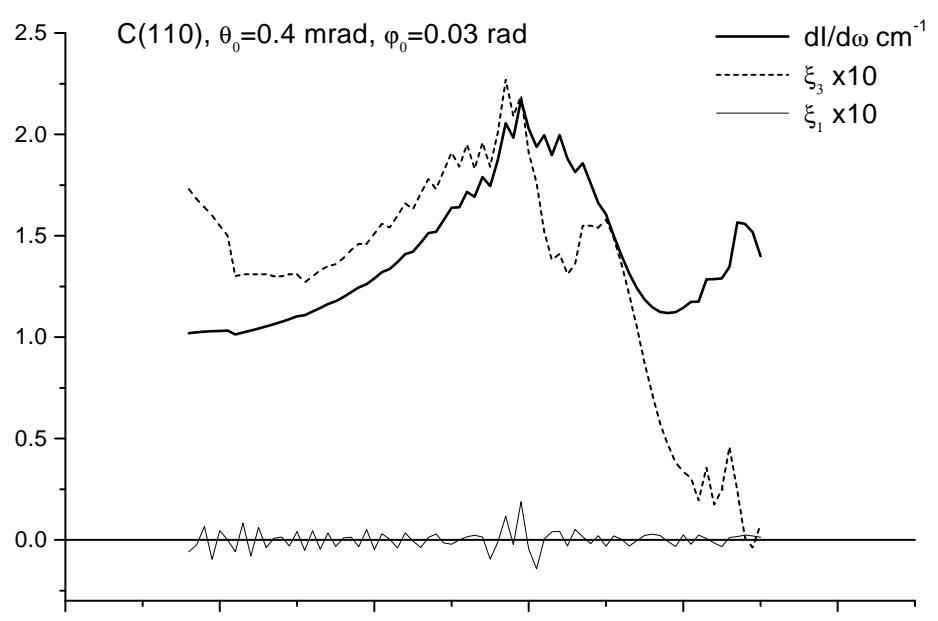
pers.

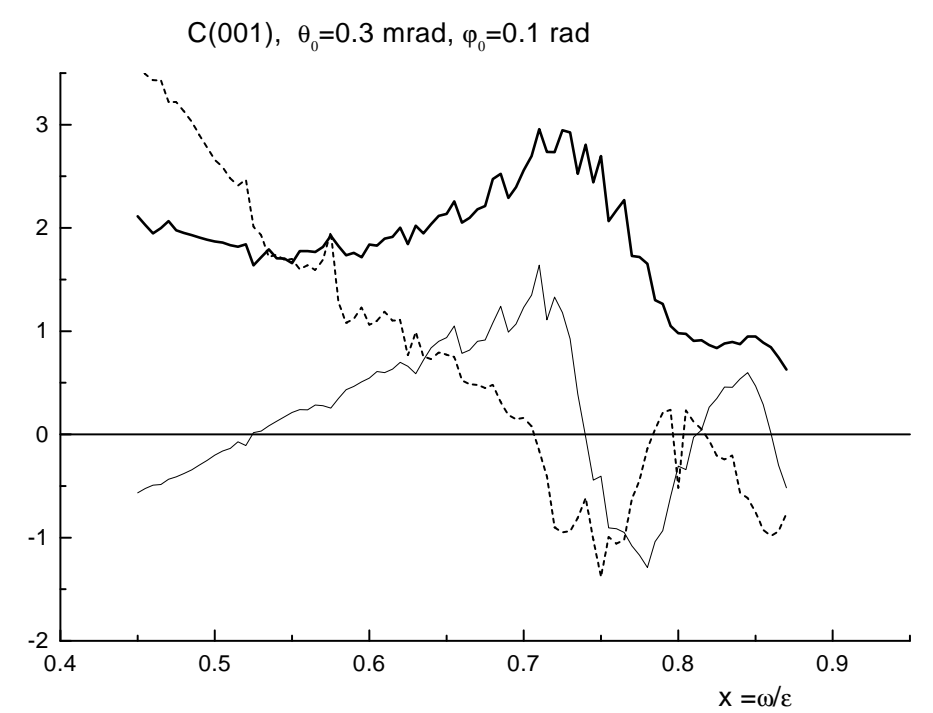

Fig.3: The spectral distribution of intensity and Stocks parameters in the range of high-energy photon peaks for $<001>$ and $<110>$ oriented diamond single crystal at $\varepsilon=150 \mathrm{Gev}$. 
This work was supported by the ISTC grant, project A-099. One of us (N.T) is grateful to Prof. R.Avakian for suggestion to participate in this project.

\section{References}

[1] M.L. Ter-Mikaelyan, High Energy Electromagnetic Processes on Condensed Media, Wiley-Intercience, New-York,1972.

[2] V.N.Bayer, V.M. Katkov and V.M. Strakhavenko, Electromagnetic Processes in Oriented Single Crystals at High Energy (in Russion), Nauka, Novosibirsk,1989.

[3] V.N.Bayer, V.M. Katkov and V.M. Strakhavenko, Nucl.Instr. Methods. B69,(1992) 25.

[4] Yu.V.Kononets, I.S. Tupitsyn, Pisma Zh. Eksp. Teor. Fiz. 59, 491-497 (1994) 148-153.

[5] V.M. Strakhovenko, Constructive Method for Calculation of Photon Emission and Pair Production Probabilities in Crystals, Budker INP 97-99, Novosibirsk, 1997.

[6] R. Mendenwaldt, S.P. Møller, B.N. Jensen et. al Phys. Lett. B281,153 (1992).

[7] A. Belkacen, Bologna, M. Chevallier, et al.,Phys.Rev.Lett. 58, 1196 (1987).

[8] J. Lindhard, Kgl. Danske Videnscab. Selsk., Mat-Fis. Medd. 34, 14 (1965). 